sich auf die Möglichkeiten der Schaffung gröBerer Flexibilität und Anpassungsfähigkeit der Wirtschaft in einem globalisierten Wettbewerb etwa durch steuer-, industrie-, innovations-, forschungs- oder regionalpolitische Instrumente beziehen. Gleiches gilt für die Frage umweltpolitischer Erfordernisse in Reaktion auf eintretende Belastungen und für die Suche nach geeigneten Möglichkeiten, um mit sozial-, arbeitsmarkt- oder auch bildungspolitischen Ansätzen bzw. mit Instrumenten der Außenwirtschaftsförderung den Globalisierungsprozess in gewünschter Weise beeinflussen zu können.

Hinsichtlich der internationalen Ebene sollen sich die Untersuchungen und Vorschläge auf drei Themenbereiche konzentrieren: erstens die Konstruktion eines internationalen Ordnungsrahmens für in besonderem Maße globalisierte Bereiche mit Hilfe der Handels-, Wettbewerbs-, Steuer- oder Investitionspolitik, der Regulierung der internationalen Finanzmärkte oder internationaler Arbeits-, Umwelt- oder Gesundheitsstandards. Besondere Bedeutung kommt hier den verschiedenen inter- und supranationalen Organen und Institutionen $\mathrm{zu}$ (WTO, Weltbank, IWF, UNO, OECD usw.). Zweitens wird es um Möglichkeiten einer effektiveren Koordinierung der internationalen Wirtschafts-, Finanz- und Geldpolitik gehen. Drittens soll schließlich die Struktur der weltwirtschaftlichen Wertschöpfungsketten ins Blickfeld genommen werden, um daraus Vorschläge für eine aus mehreren Gründen wünschenswerte stärkere Beteiligung der Entwicklungs- und Transformationsländer am weltwirtschaftlichen Beziehungsgeflecht ableiten zu können. Hierbei soll auch in Betracht gezogen werden, inwieweit sich veränderte Austauschbeziehungen positiv auf den Umwelt- und Klimaschutz sowie entsprechende Kooperationen auswirken könnten. Damit soll der Weg zur Schaffung von günstigen wirtschaftlichen, sozialen, ökologischen und politischen Rahmenbedingungen auf globaler Ebene erleichtert werden.

\section{Dokumentation 2000 der TA-Akademie bald im Internet}

\author{
von Manfred Rohr, Akademie für Technik- \\ folgenabschätzung in Baden-Württemberg
}

\begin{abstract}
Mit der Dokumentation 2000 „Technikfolgenforschung in Baden-Württemberg" hat die TA-Akademie die vierte Ausgabe ihres landesweiten Nachschlagewerkes im Bereich der Technikfolgenforschung und Technikfolgenabschätzung (TA) vorgelegt. Durch die Vernetzung von Forschungsgebieten und regionalen Forschungskapazitäten sollen Synergieeffekte besser genutzt und der innovationsorientierte Ideen- und Wissenstransfer im TA-Netzwerk BadenWürttemberg und darüber hinaus beschleunigt werden. Um einem breiteren Nutzerkreis aus Wissenschaft und Wirtschaft sowie aus Politik, Verwaltung und interessierter Öffentlichkeit einen schnelleren und zugleich leichteren Wissenszugang im Kontext von Technikfolgenforschung, -abschätzung und -gestaltung zu ermöglichen, wird von der TA-Akademie bis Jahresende die Online-Verfügbarkeit der Dokumentation im Internet als neue Informations- und Kommunikationsplattform „TANet-BW" realisiert.
\end{abstract}

\section{Entwicklung und Gestaltung der Dokumen- tation}

Wie durch die 1347 dokumentierten Forschungsprojekte von 400 Einrichtungen belegt wird, haben die Aktivitäten im Bereich der Technikfolgenforschung in Baden-Württemberg in den letzten Jahren weiter zugenommen und sind thematisch vielfältiger geworden; das TA-Netzwerk Baden-Württemberg hat an Dichte und Leistungsfähigkeit deutlich zugenommen - das geht aus der letztjährigen Umfrage der TA-Akademie zur Aktualisierung der gleichnamigen Dokumentation hervor. Ob in Universitäten, Fachhochschulen, Großforschungseinrichtungen oder anderen wissenschaftlichen Einrichtungen - immer mehr Wissenschaftler, Forscher und Entwickler der verschiedensten Disziplinen kooperieren erfolgreich miteinander und berücksichtigen in ihren Arbeiten die Wechselwirkungen mit anderen 
Fachdisziplinen und die möglichen Auswirkungen auf unterschiedliche Lebensbereiche.

Naturwissenschaftler erforschen den nachhaltigen Umgang mit den natürlichen Ressourcen Wasser, Boden und Luft. Ingenieure vergleichen Abfallbehandlungsverfahren und bewerten deren Umweltverträglichkeit. Mediziner und Gesundheitsökonomen evaluieren medizinische Verfahren und Technologien. Politologen untersuchen den Zusammenhang von Bevölkerungswachstum und zunehmenden Umweltproblemen, Sozialwissenschaftler den Wandel der Arbeitswelt. Für eine praktische Umsetzbarkeit der Forschungsergebnisse in nachhaltige und zukunftsfähige Problemlösungen sind dabei die Betrachtung der Potenziale und Chancen des Einsatzes von Technik ebenso von Bedeutung wie die Abschätzung und Bewertung der mit ihrer Anwendung verbundenen Risiken und die Bewältigung damit zusammenhängender möglicher Konflikte und Akzeptanzprobleme in der Bevölkerung.

Bei der Gestaltung der Dokumentation 2000 wurde im Hinblick auf größtmögliche Nutzerfreundlichkeit und Funktionalität zunächst einmal die bewährte Form der vorhergehenden Ausgabe zugrunde gelegt und deshalb wieder eine Kombination aus einer Broschüre der Institutionen und einer elektronischen Gesamtausgabe einschließlich aller aufgenommenen Projekte auf CD-ROM gewählt, die der Dokumentation beiliegt. Das inhaltliche Konzept der Gesamtdokumentation und ihre Dreiteilung in Institutionen, Projekte und Register wurde ebenso beibehalten wie die Strukturierung des Projektteils in die bekannten 13 Themenfelder.

Durch die Broschüre der Institutionen wird dem Nutzer ein schneller Überblick über das TA-Netzwerk Baden-Württemberg ermöglicht, entsprechende Register sind bei der Orientierung und Suche nach Institutionen, Personen und Sachwörtern behilflich. Mit der auf der CD-ROM befindlichen Datenbank kann neben den Institutionen auch der umfangreiche Projektteil der Gesamtdokumentation schnell und direkt erschlossen werden. Entsprechende Suchroutinen über Sachwörter oder eine Freitextsuche ermöglichen dem Nutzer schnelle Recherchen sowohl im Institutionenteil als auch im Projektteil der Dokumentation. Besonders hilfreich für alle Internetnutzer ist die
Möglichkeit, dass man von der CD-ROM aus unmittelbaren Zugang auf die Homepages der dokumentierten Einrichtungen erhält und sich so über den Dokumentationsinhalt hinaus noch weitergehend informieren kann. Außerdem besteht per E-Mail eine direkte Kommunikationsmöglichkeit zu den dokumentierten Personen.

Als erweiterte Ausbaustufe wird bis Ende des Jahres die Online-Verfügbarkeit der Dokumentation 2000 im Internet unter der Adresse www.ta-net-bw.de als neue Informationsund Kommunikationsplattform ,TA-Net-BW“ (Abb. 1) realisiert, um somit einem noch breiteren Nutzerkreis aus Wissenschaft und Wirtschaft sowie aus Politik, Verwaltung und interessierter Öffentlichkeit einen schnelleren und zugleich leichteren Wissenszugang im Kontext von Technikfolgenforschung, -abschätzung und -gestaltung zu ermöglichen. Die leistungsfähige und nutzerfreundliche Plattform soll dazu beitragen, die fachübergreifende Kooperation zwischen den Einrichtungen durch gezielte Information und die Vermittlung kompetenter Ansprechpartner zu fördern und damit die Forschungsinfrastruktur des Landes weiter zu verbessern und insgesamt effektiver zu gestalten.

Diese als Transfer- und Vermittlungsstelle für TA-Informationen im weitesten Sinne konzipierte Plattform ist schwerpunktmäßig auf die Unterstützung von Forschungsaktivitäten und -kapazitäten im TA-Netzwerk Baden-Württemberg ausgerichtet, wird aber darüber hinaus auch landesübergreifend mit anderen Forschungs- und Innovationsnetzwerken sowie mit Fach- und Forschungsdatenbanken korrespondieren.

\section{Ergebnisse der Umfrage}

Seit der Erstausgabe der Dokumentation im Jahr 1993 konnte die Zahl der dokumentierten Institutionen kontinuierlich gesteigert werden und hat sich inzwischen mehr als verdoppelt, die Zahl der Forschungsprojekte sogar mehr als verdreifacht (Abb. 2). 
Abb. 1: TA-Netzwerk Baden-Württemberg (TA-Net-BW)

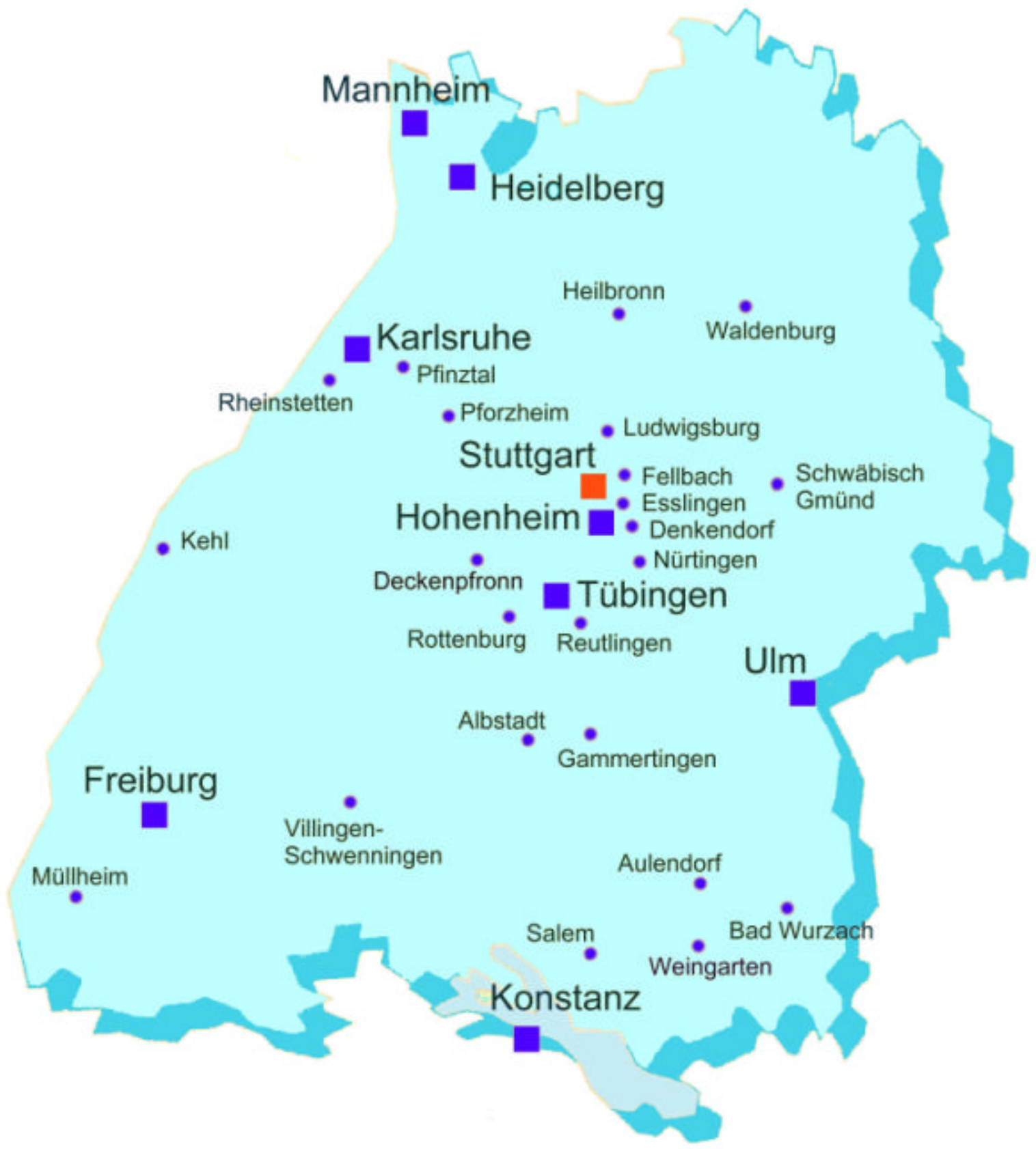

TA-Akademie, Universität und andere Institutionen

Universitätszentren und andere Institutionen

- Institutionen im außeruniversitären Bereich 


\section{Abb. 2: Entwicklung der Dokumentation}

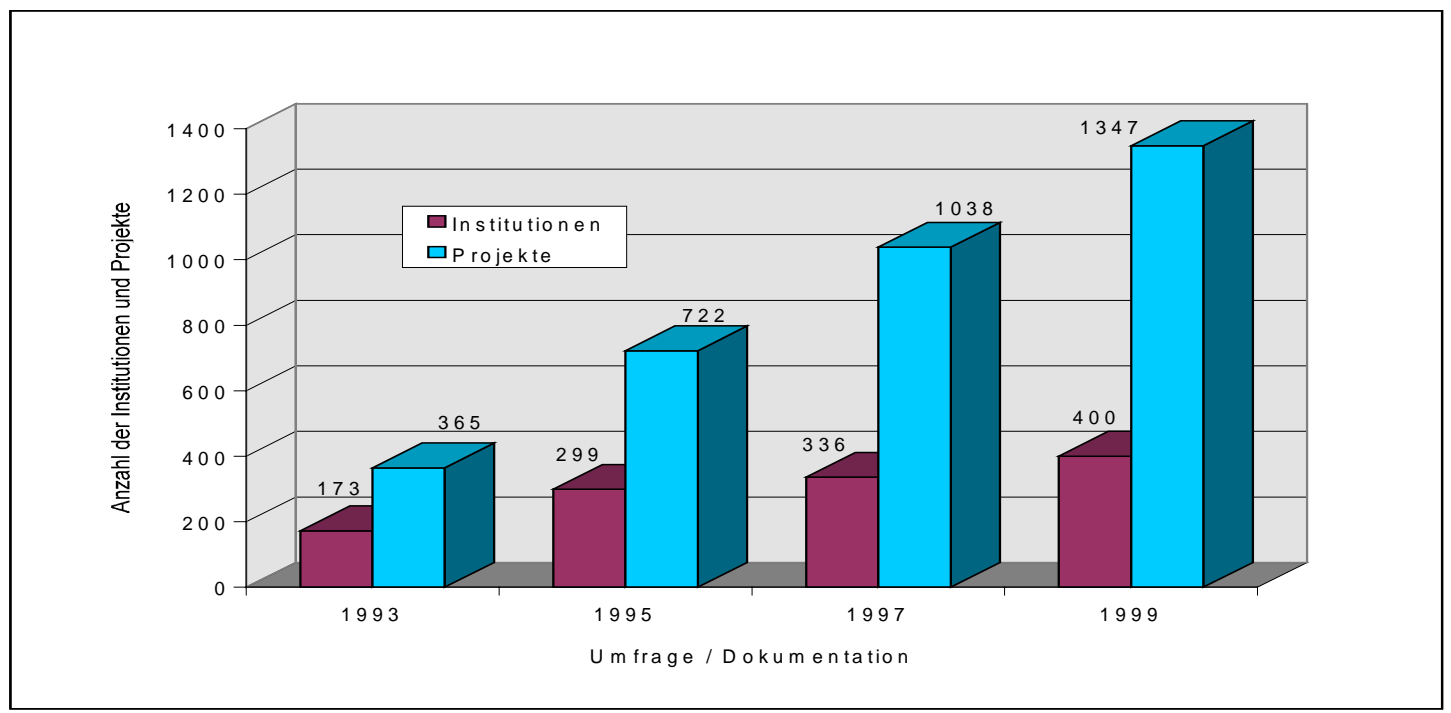

Wie die Umfrageergebnisse zeigen, konzentriert sich das TA-Forschungspotenzial in Baden-Württemberg mit ca. $66 \%$ nach wie vor im universitären Bereich und hier schwerpunktmäßig in den naturwissenschaftlich-technisch ausgerichteten Universitäten Karlsruhe, Stuttgart, Freiburg und Hohenheim. Rund $8 \%$ der Institutionen entfallen auf Großforschungseinrichtungen wie das Forschungszentrum Karlsruhe, die Fraunhofer-Gesellschaft sowie das Deutsche Zentrum für Luft- und Raumfahrt (DLR). Der Anteil der Institutionen und Einrichtungen aus dem Bereich der Fachhoch- schulen und Pädagogischen Hochschulen beträgt etwa $6 \%$. Das restliche Forschungspotenzial (ca. $20 \%$ ) entfällt auf gemeinnützige und private Einrichtungen (Abb. 3).

Wie aus der Verteilung der Projekte auf die Themenfelder hervorgeht, entfallen die meisten Projekte auf die Themenfelder

- Umwelt sowie

- Wirtschaft / Nachhaltige Entwicklung.

Etwa die Hälfte der Projekte (711) entfällt schwerpunktmäßig auf folgende Themenfelder:

\section{Abb. 3: Verteilung der Institutionen und Projekte}

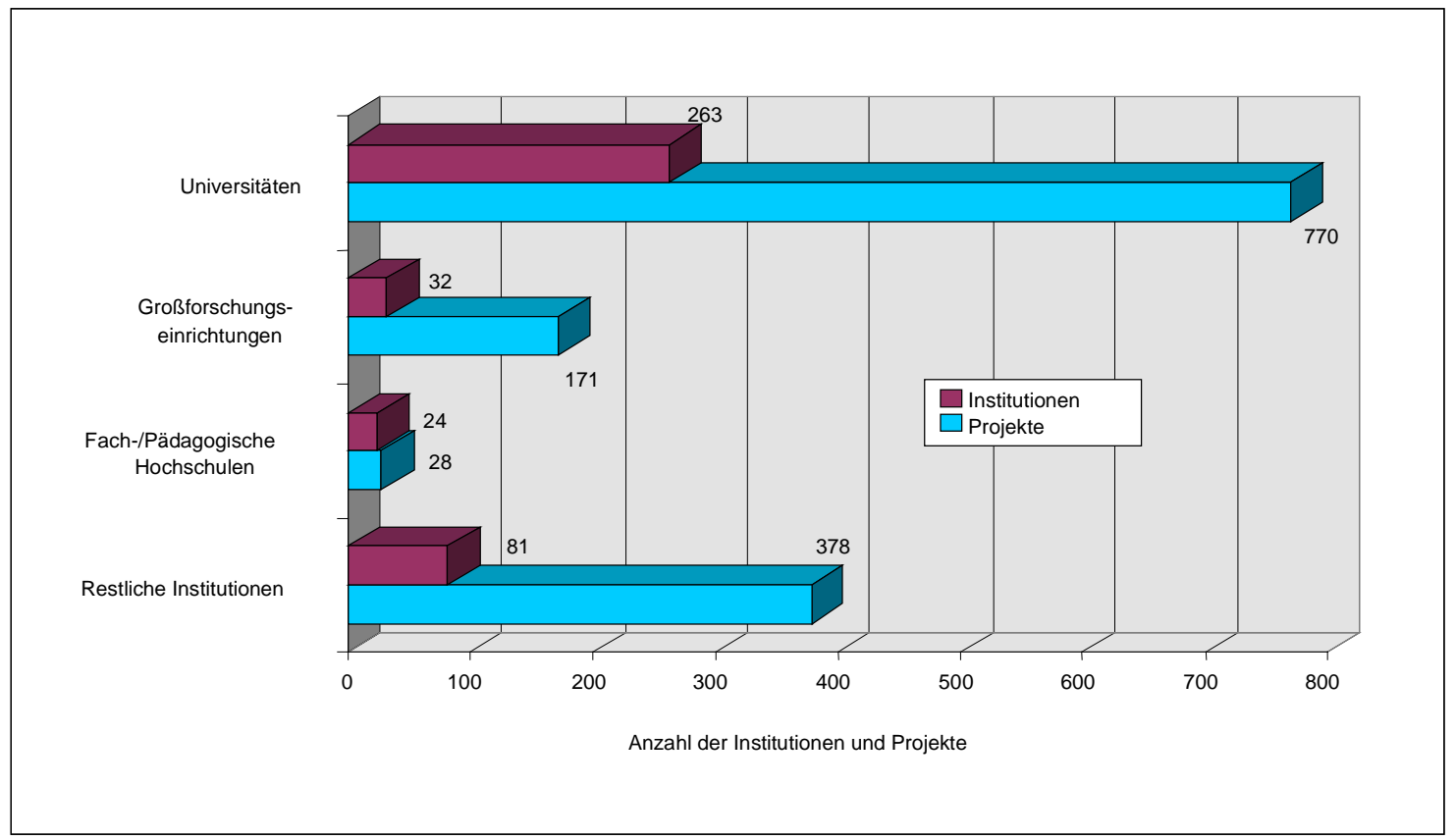


Abb. 4: Verteilung der Projekte auf die Themenfelder

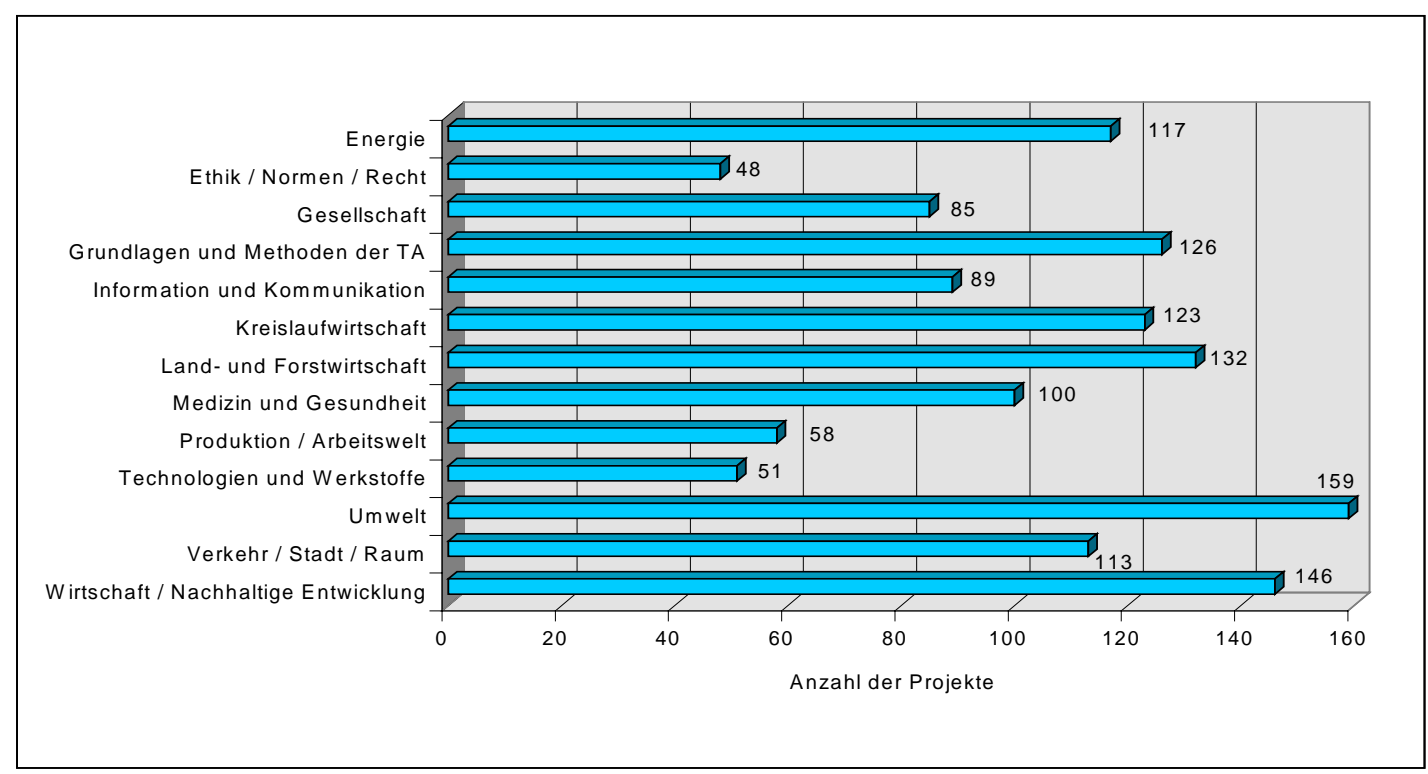

- $\quad$ Land- und Forstwirtschaft

- Grundlagen und Methoden der TA

- Kreislaufwirtschaf

- Energie

- Verkehr / Stadt / Raum

- Medizin und Gesundheit (Abb. 4).

Auf Anfrage der TA-Akademie wurde die Umfrage von einer Reihe von Institutionen und Einrichtungen wiederum dazu genutzt, um auf Gebiete und Themenfelder hinzuweisen, in denen ihrer Meinung nach noch Forschungsdefizite bestehen. Die hierzu bei der TA-Akademie eingegangenen zahlreichen Hinweise, Anregungen und Vorschläge zu möglichem Forschungs- bzw. Handlungsbedarf werden ausgewertet und in ausgewählten Schwerpunktrichtungen thematisiert. Über die Ergebnisse wird an anderer Stelle gesondert berichtet.

Die Dokumentation 2000 (incl. CD-ROM) kann gegen eine Schutzgebühr von DM 30,-bei der TA-Akademie unter der ISBN-Nr. 3-934629-03-2 bestellt werden (Fax: + 49 (0) 711/9063-299).

\section{Kontakt}

Dr.-Ing. Manfred Rohr

Akademie für Technikfolgenabschätzung

in Baden-Württemberg

Industriestraße 5, D-70565 Stuttgart-Vaihingen

Tel.: + 49 (0) 711 / 9063 - 103

E-Mail: $\underline{\text { manfred.rohr@ta-akademie.de }}$

\section{The Centre for Energy Policy and Economics - A new TA- Institution in Switzerland}

\author{
by Eberhard Jochem, Centre for Energy \\ Policy and Economics
}

The Centre for Energy Politics and Economics (CEPE) is a newly established research centre in Zürich. Its goal is to extend the scientific and technical research work of the Swiss Federal Institutes of Technology in Zurich and Lausanne and the Paul Scherrer Institute (PSI) to include the interface between technology, economy and society. By its analyses of energy economics and policy issues, CEPE also strives to improve the decision-making processes in business and government.

\section{Mission}

The Centre for Energy Policy and Economics (CEPE) was established in 1999 in order to extend the scientific and technical research areas of the Swiss Federal Institutes of Technology in Zurich and Lausanne and the Paul Scherrer Institute (PSI) to include the interface between technology, economy and society. Through its national and international interdisciplinary research and advisory activities, CEPE seeks to point out in a timely manner 